\title{
Management of Hyperemesis Gravidarum
}

\author{
Francis Ajang Magaji $M B, B S, F M C O G$ (Nig), FWACS. \\ Department of Obstetrics and Gynaecology, Jos University Teaching Hospital, Jos, Nigeria.
}

\begin{abstract}
Key words: Hyperemesis gravidarum; hyperemesis, hCG; Human chorionic gonadotropin, ALT; Alanine amino trasferases, AST; Aspartate amino transferases.
\end{abstract}

\section{Introduction}

This is one of the most common of all the tribulations of the first trimester and it is characterized by nausea and vomiting which in severe cases may lead to dehydration and require hospitalization.

One definition of hyperemesis that has been proposed is persistent vomiting, weight loss exceeding $5 \%$ of prepregnancy body weight, and ketonuria. ${ }^{1}$ It may be because of the differences in the definition of the disease that the true incidence is not well documented. The disease is said to vary from as low as 3 cases per 1000 pregnancies to as high as 1 in $100 .^{2-4}$

Hyperemesis may be considered as the severe end of the spectrum of morning sickness; with no clear demarcation between the common and pathologic forms. Some degree of nausea and vomiting occur in 50 to $90 \%$ of all pregnancies. These symptoms have a mean onset at five to six weeks of gestation, peak at nine weeks, and usually abate by sixteen to eighteen weeks of gestation. ${ }^{5}$ The discomfort may occur at any time of the day and often $(80 \%)$ persist throughout the day. In 15\% gravida, nausea and vomiting continue until term and in $5 \%$ of cases until delivery. ${ }^{6}$

\section{Risk Factors}

Our ignorance of the cause is almost as striking as the success which nearly always attends treatment. The condition is commoner in women with high body weight, nulliparity and multiple gesteations. It has also been associated with trophoblastic disease by $8 \%{ }^{8}$ In addition, non pregnant women who experience nausea and vomiting after estrogen exposure, previous hyperemesis gravidarum, motion sickness, or with exposure to certain tastes (supertasters) are more likely to have pregnancy related nausea and vomiting.

Advanced maternal age (35 years) and Cigarette smoking (because of the nicotine) were protective in one report.

\section{AetioPathogenesis}

Hyperemesis has been attributed to a number of factors, but the aetiology remains uncertain.
Theories advanced to explain hyperemesis are supported in some studies while others have found discordant results. The following categories represent the predominant theories considered in the pathogenesis of hyperemesis gravidarum:

Psychologic factors have been proposed to contribute to the pathogenesis of hyperemesis. However, no definitive study has shown that the psychologic make up of patients with hyperemesis gravidarum differs from those without the disease.

Hormonal theory; no single hormonal profile can accurately predict the presence of hyperemesis gravidarum. Elevated serum concentrations of estrogen and progesterone have long been implicated in the pathogenesis of this disorder. Although several lines of evidence support a role, the fact that sex hormone levels peak in the third trimester when symptoms of hyperemesis have long resolved disputes this association. ${ }^{9}$ In comparison to six hormones, serum concentrations of hCG levels peak during the first trimester, the time at which hyperemesis gravidarum is typically seen. The observation that serum hCG is higher in women with hyperemesis than in other pregnant women is therefore compatible with a possible aetiologic role for this hormone. ${ }^{10,11}$ However, an association between hCG levels and hyperemesis gravidarum has not been clearly established. There are many who consider that hyperemesis is a manifestation of allergy, and found large quantities of histamine in urine of such patients. It is also shown that adrenocortical insufficiency causes hypersensitivity to histamine and favours the development of allergic manifestations.

Gastric Motility may be abnormal in hyperemesis gavidarum. However, studies addressing motility disturbance have shown conflicting results, suggesting that these abnormalities are not highly predictive of the disease.

Several other theories to explain hyperemesis have been suggested, including specific nutrient deficiencies (e.g., zinc), alterations in lipid levels, changes in the autonomic nervous system and infection with Helicobacter pylori. ${ }^{12,13}$

None of the above theories is consistently associated or highly predictive of the disease. 


\section{Clinical Features}

Hyperemesis presents early in the first trimester of pregnancy, usually, in the fourth to tenth week of gestation, with intractable vomiting and associated ptyalism (excessive salivation).

With deteriorating condition, the tongue becomes dry and furred while the breath smells strongly of acetone. Her pulse rate starts to rise and blood pressure falls. Many patients have weight loss exceeding 5\% of their body weight and epigastric tenderness is frequently noted.

In the later stages of the disease, temperature starts to rise, jaundice supervenes and haematemesis may occur. Squint, diplopia and nystagmus maybe noted and retinal haemorrhages appear.

Various palsies develop and there may be great tenderness in the legs together with other signs of peripheral neuritis. Ultimately, Wernicke's encephalopathy becomes fully established and the patient usually dies in coma.

Other conditions unrelated to pregnancy that can cause persistent nausea and vomiting include gastro intestinal disorders (e.g., hepatitis, pancreatitis, cholelithiasis), genitourinary tract disorders, metabolic (e.g., diabetes, porphyria), and neurological disease (e.g., migraine, tumor, vestibular lesions), drug toxicity, hiatus hernia and psychological problems.

Nausea and vomiting that develop after ten weeks of gestation are not likely due to hyperemesis. Preeclampsia, HELLP syndrome, and fatty liver of pregnancy can also cause nausea and Vomiting typically in the later half of pregnancy. These differentials are associated with symptoms which include abdominal pain, fever, headache and hypertension.

Goiter may also cause nausea and vomiting, features that distinguish the transient hyperthyroidism of hyperemesis from hyperthyroidism of other causes (mostly Graves disease) are the vomiting, absence of goiter and ophthalmopathy, and absence of the common symptoms and signs of hyperthyroidism (heat intolerance, muscle weakness, tremor).

Similarly, liver enzyme elevations are 5 to 10 times greater in hyperemesis than pancreatitis.

\section{Investigations}

The urine is usually concentrated and in addition to acetone, it may contain albumin and bile with chlorides greatly diminished or absent. There is often an increased in haematocrit, indicating haemoconcentration may be underestimated unless the decline in haematocrit seen in normal pregnancies is considered.

Patients may present with electrolyte derangements such as hypokalaemia and metabolic alkalosis. Abnormal liver enzymes may be noted in approximately $50 \%$ of patients who are hospitalized with hyperemesis ${ }^{4}$. The most stricking abnormality is an increase in serum amino transferases. ALT is typically elevated in greater proportion to AST. Values for both maybe in the low hundreds and rarely as high as $1000_{\mathrm{U}} / \mathrm{L}$. Hyperbilirubinaemia also can occur, but rarely exceeds $4 \mathrm{mg} /{ }_{\mathrm{L}}{ }^{15}$ The degree of these abnormalities in liver tests correlates with the vomiting; the highest elevations are seen in patients with the most severed or protracted vomiting. Abnormal liver biochemical tests resolve promptly upon resolution of the vomiting.

Mild hyperthyroidism maybe associated with hyperemisis, perhaps due to higher serum concentration of hCG which may have more thyroid-stimulating activity. ${ }^{10}$ One report found low serum TSH concentration more often in women with hyperemesis than in normal pregnant women (60 vs. 9\%). ${ }^{11}$ Some of these women had elevated serum free $\mathrm{T}_{4}$ concentration and therefore had hyperthyroidism. In addition, serums free $\mathrm{T} 4$ concentrations are only minimally elevated in women with hyperemesis, where as both are usually unequivocally elevated in pregnant women with true hyperthyroidism.

Hyperparathyroidism has been described in pregnancy and associated with hyperemesis. ${ }^{16,17}$ While uncommon, it should be considered in patients with hypercalcemia. In such patients, the hypercalcemia may contribute to the vomiting.

Given the characteristic symptoms of hyperemesis, a liver biopsy is rarely needed to exclude other causes for the laboratory findings. When liver biopsy has been performed, it has been either normal or shown non specific findings. Inflammation is absent, but steatosis. Certrilobular vacuolization, cell dropout, and rare bile plugs maybe seen. ${ }^{15,18,19}$

\section{Treatment}

Symptoms usually resolve by the $18^{\text {th }}$ week of gestation, regardless of therapy. Many patients respond to rehydration and a short period of gut rest, followed by reintroduction of oral feeding. Further therapy in selected patients may include antiemetic, corticosteroids and enteral and parenteral nutrition.

Nonpharmacological: The cornerstone here is avoidance of environmental triggers ${ }^{20}$. Example of 
some triggers includes: stuffy rooms, odors (eg, perfume, chemicals, food and smoke), heat, humidity, noise, and visual or physical motion (eg, flickering lights, driving). ${ }^{21}$ Brushing teeth after eating, quickly changing position and not getting enough rest may also aggravate symptoms.

Supplements containing irons should be avoided until symptoms resolve. Dietary manipulations such as frequent and high carbohydrate, eliminating fatty or spicy foods or eating salty or high protein snacks/meals appear to help some weomen. ${ }^{22}$ Fluids (cold, clear carbonated or sour) may be tolerated better if taken in small amounts between meals. ${ }^{5}$ Aromatic therapies including lemon (lemonade), mint (tea), or orange have also been described as useful. Women with hyperemesis are encouraged to eat before or as soon as they feel hungry in order to avoid an empty stomach that may aggravate nausea. ${ }^{23}$

Other options are acupuncture or acupressure wrist bands and powdered ginger. ${ }^{24}$ Although acupuncture has been shown to be useful when compared to no treatment, there appears to be a strong placebo effect. ${ }^{25-27}$ Hypnosis has been reported to be helpful in selected patients. ${ }^{28}$ Psychotherapy can be a useful adjunctive therapy, particularly if psychological sources of anxiety are indentified and can be ameliorated. ${ }^{29,30}$

Nutritional: Nutritional status and methods of alimentation (e.g., tube feedings, parenteral nutrition) should be assessed in conjunction with a nutritionist or nutrition service. Rare patients with protracted vomiting require parenteral nutrition. Enteral nutrition via gastric or duodenal intubation may also be beneficial and preferable to the parenteral route. In one report, enteral nutrition also relieved the nausea and vomiting. ${ }^{32}$ It is important to pay close attention to replenishing vitamins, electrolytes and minerals such as magnesium, phosphorous, and potassium. ${ }^{32}$ Thiamine supplementation (100mg IV daily for 2 to 3 days) is recommended for women who have vomiting for more than three weeks. ${ }^{5}$ Patients who have not eaten for several days may develop oedema when resuming feeding with carbohydrates. ${ }^{33}$ This results from the retention of sodium during fasting combined with enhanced sodium resorption due to the actions of insulin once carbohydrates are reintroduced. ${ }^{34}$

Dehydration should be corrected with intravenous fluid solution, such as Lactated Ringers supplemented with appropriate electrolytes and vitamins. Relief of symptoms is common within one to two days of rehydration. ${ }^{5}$ Hospitalization, as well as replenishment of fluid and electrolytes, may contribute to palliation of symptoms.

Pharmacological: There is paucity of evidence base data from which to recommend a treatment plan. Thus, a reasonable approach is to begin therapy with agents that have minimal maternal side effects and, if these are ineffective, substitute other drugs in a step-wise progression.

Pyridoxine (vitamin $\mathrm{B}_{6}$ ): 10 to $25 \mathrm{mg}$ orally TID may help women with mild to moderate nausea and minimal vomiting. ${ }^{24}$

Antiemetics, including promethazin (phenergan 12.5 to $25 \mathrm{mg}$ Q4 h PO IM, or PR), metoclopromide (5 to $10 \mathrm{mg}$ Q8 h IV or PO), Ondansetron (8mg Q12 $\mathrm{h}$ IM or PO), prochlorperazine (5 to $10 \mathrm{mg} \mathrm{Q} 3$ to $4 \mathrm{~h}$ IM or PO or $25 \mathrm{mg}$ BID PR), and droperidol combined with diphenhydramine, may benefit selected patients. ${ }^{35,36}$

Corticosteroids, which can be safely given during pregnancy, have been used successfully in women with severe and refractory hyperemesis, although the mechanism of action is not well understood. ${ }^{37-40}$

Surgical: It use to be the practice that, patients that fail to respond to the above energetic treatment, therapeutic abortion was considered as last option, especially in multiparae, in whom vomiting often has a more sinister significance. Other indications for therapeutic abortion were: Jaundice, persistent albuminuria, polyneuritis and neurological signs, temperature constantly above 100.4 and a pulse rate remaining above 100 . The outcome following therapeutic abortion was relatively poor because the decision to operate is easily too long delayed, in which case death maybe precipitated rather than prevented. $^{41}$

It is nowadays so rarely that pregnancy has to be interrupted that one is very naturally reluctant to take such drastic action but to operate too late is just as bad as not operating at all.

\section{Outcome}

Changes in weight: Weight gain in early pregnancy is lower in women with mild to moderate vomiting, however, total weight gain during the entire pregnancy is similar to that in women with no vomiting or only nausea. ${ }^{42}$ In contrast, women with hyperemesis who require multiple hospitalizations may not have "Catch up" weight gain. ${ }^{43}$ An adverse effect on birth weight is more likely in these women. ${ }^{2}$ 
Spontaneous miscarriage: the rate of miscarriage is decreased. In one meta-analysis, the odds ratio for miscarriage in women with nausea and vomiting in the first 20 weeks of pregnancy was $0.36(95 \%$, CI 0.2 to 0.42$){ }^{44}$

Birth defects: There is no clear increase in the risk of birth defects among offspring of gravida with hyperemesis, whether or not they take antiemetic medications. ${ }^{2,45,46}$

A number of reports have demonstrated that antiemetic drug therapy has no effect on the incidence of congenital anomalies. ${ }^{45}$ Data from a Swedish registry on women who took promethazine or meclizine in the first trimester did not show an increase in the rate of birth defects. ${ }^{46}$ Another series found a lower rate of congenital heart disease in infants of mothers who took antiemetics in the first two months of pregnancy. ${ }^{45}$ Finally, a meta-analysis that examined the association between antihistamine use and major malformations suggested that $\mathrm{H}_{1}$ - receptor blockers have a protective effect (odds ratio 0.76 ). ${ }^{47}$

\section{Conclusion}

Women with mild nausea and vomiting during pregnancy have better pregnancy outcomes than women without these symptoms. These women experience fever miscarriages and stillbirths. ${ }^{5,42}$ By comparison, hyperemesis gravidarum led to significant morbidity and even mortality in the past. In the modern era, the availability of intravenous fluids has greatly reduced morbidity, and mortality is unusual.

Despite the severity of the illness and its attendant weight loss, there is no difference in birth weight, gestational age, or birth defects between affected pregnancies and those unaffected by hyperemesis gavidarum. ${ }^{3,4}$ The disease may recur uncommonly during subsequent pregnancies.

\section{References}

1. Goodwin, TM. Hyperemesis graviderum. Clin Obstet Gynecol 1998; 41:597.

2. Kallen, B. hyperemesis during pregnancy and delivery outcome: A registry study. Eur J Obstet Gynecol Reprod Boil 1987; 26:292.

3. Hallak, M, Tsalamandris, $\mathrm{K}$, Dombrowski, M. hyperemesis graviderum. Effects on fetal outcome. J Reprod med 1996; 41:871.

4. Tsang, IS, Katz, VL, Wells, SD. Maternal and fetal outcomes in hyperemesis graviderum. Int J Gynaecol Obstet 1996; 55:231.

5. Association of professors of Gynaecology and Obstetrics. Nausea and vomiting of pregnancy. Association of professors of Gynaecology and Obstetrics, Washington, DC 200.
6. Tierson, FD, Olsen, CL, Hook, EB. Nausea and vomiting of pregnancy and association with pregnancy outcome. Am J Obstet Gynecol 1986; 155:1017.

7. Klebanoff, MA, Koslowe, PA, Kaslow, R. Epidemiology of vomiting in early pregnancy. Obstet Gynocl 1985; 66:612.

8. Soto-Wright, V, Benrstein, M, Goldstein, DP. The changing clinical presentation of complete molar pregnancy. Obstet Gynecol 1995; 86:775.

9. O'Leary,P, Boyne, P, Flett, P. Longitudinal assessment of changes in reproductive hormones during normal pregnancy, clin chem 1991; 37:667.

10. Kimura, M, Mino, N, Tameki, H. Gestational thyrotoxicosis and hyperemesis gaviderum: possible role of hCG with higher stimulating activity clin endocrinol 1993; 38:345.

11. Goodwin, TM, Montoro, M, Mestman, JH. The role of chorionic gonadotropin in transient hyperthyroidism of hyperemesis graviderum. J clin Endocrinol Metab 1992; 75:1333.

12. Frigo,P, Lang, C, Reisenberger, K. Hyperemesis gravidarum associated with Helicobacter pylori seropositivity. Obstet Gynecol 1998; 19:615.

13. Jacoby, EB, Porter, KB. Helicobacter pylori infection and persistent hyperemesis gravidarum. Am J perinatol $1999 ; 16: 85$.

14. Abell, T, Riely, C. hyperemesis gavidarum. Gastroenterol Clin North Am 1992; 21:835.

15. Larrey, D, Rueff, B, Feldmann, G. Recurrent Jaundice caused by recurrent hyperemesis gravidarum. Gut $1984 ; 25: 1414$.

16. Budd, DC, Kumka, MM, suda, AK, Fink, DL. Hyperthyroidism masquerading as hyperemesis gravidarum. NJ Med 1988; 85:811.

17. Pederson, NT, Permin, H. Hyperparathyroidism and pregnancy. Acta Obstet Gynecol scant 1975; 54:282.

18. Abell, T. Nausea and vomiting of pregnancy and the electrogastrogram: old disease, new technology. Am J Gastroenterol 1992; 87:689.

19. Adams, RH, Gordon, J, Combes, B. Hyperemesis graviderum: Evidence of hepatic dysfunction. Obstet Gynecol 1968; 31:659.

20. Erick, M. No more morning sickness. A survival Guide for pregnant women. Plume, New York 1993.

21. Erick, M. Hyperolfaction and hyperemesis gravidarum: What is the relationship? Nutr Rev 1995; 52:289.

22. Jednak, MA, Shadigian, EM, Kim, MS. Proteint meals reduce nausea and Gastric slow wave dysrhythnic activity in first trimester pregnancy. Am J physiol 1999; 277:G855.

23. Newman, V, Fullerton, JT, Anderson, PO. Clinical advances in the management of severe nausea and vomiting during pregnancy. J Obstet Gynecol Neonatal Nurs 1993; 22:483.

24. Jewell, D, Young, G. Interventions for nausea and vomiting in early pregnancy. Cochrane Database syst Rev 2000;: CD 000145.

25. Werntoft, E, Dykes, AK. Effect of acupressure on nausea and vomiting during pregnancy. A randomized, placebo-controlled, pilot study. J Reprod Med 2001; 46:835.

26. Knight, B, Mudge, C, Openshaw. Effect of acupuncture on nausea of pregnancy: a randomized, controlled trial. Obstet Gynecol 2001; 97:184. 
27. Dundee, JW, Sourial, FB, Ghaly, RG, Bell, PF. P6 acupressure reduces morning sickness. J R soc Med 1988; 81:456.

28. Simon, EP, Schwartz, J. Medical hypnosis for hyperemesis gravidarum. Birth 1999; 26:248.

29. Henker, FO $3^{\text {rd }}$. Psychotherapy as adjunct in treatment of vomiting during pregnancy. South Med J 1976; 69:1585.

30. Zechnich, R, Hammer, T. Brief psychotherapy for hyperemesis gravidarum. Am Fam physician 1982; 26:179.

31. Hsu, J, Clark-Glen, R, Nelson, D, Kim, C. Nasogastric enteral feeding in the management of hyperemesis gravidarum. Obstet Gynecol 1996; 88:343.

32. Brooks, MJ, Melnik, G. The refeeding syndrome: an approach to understanding its complications and preventing its occurrence. Pharmacotherapy 1995; 15:713.

33. Veverbrants, E, Arky, RA. Effects of fasting and refeeding. Studies on sodium, potassium and water excretion on a constant electrolyte and fluid intake. $\mathrm{J}$ Clin endocrinol Metab 1969; 29:55.

34. DeFronzo, RA, Cooke, CR, Andres, R. The effect of insulin on renal handling of sodium, potassium, calcium, and phosphate in man. J Clin Invest 1975; 55:845.

35. Sullivan, C, Johnson, C, Roach, H. A pilot study of intravenous aldosterone for hyperemesis gravidarum. Am J Obstet Gynecol1996; 174:1565.

36. Nageotte, MP, Briggs, GG, Towers, CV, Asrat, T. Droperidol and diphenhydramine in the management of hyperemesis gravidarum. Am J Obstet Gynecol 1996; 174:1801.

37. Safari, HR, Alsulyman, OM, Gherman, RB. Experience with oral methylprednisolone in the treatment of refractory hyperemesis gravidarum. Am J Obstet Gynecol 1998; 178:1054.
38. Taylor, R. Successful management of hyperemesis gravidarum using steroid therapy. QJM 1996; 89:103.

39. Nelson-Piercy, C, Fayers, P, de Swiet, M. Randomized, double-blind, placebo-controlled trial of corticosteroids for the treatment of hyperemesis gravidarum. BJOG 2001; 108:9.

40. Moran, P, Taylor, R. Management of hyperemesis gravidarum: the importance of weight loss as a criterion for steroid therapy. QJM 2002; 95:153.

41. Wallace, B. Vomiting, Hiatus Hernia and Heartburn. In: Ian Donald (Ed): Practical Obstetric problems Lloydluke (medical books) ltd.London. 1974; 202-207.

42. Flaxman, SM, Sherman, PW. Morning sickness: A mechanism for protecting mother and embryo. Q Rev Biol 2000; 75:113.

43. Depue, RH, Bernstein, L, Ross, RK. Hyperemesis gravidarum in relation to estradiol levels, pregnancy outcome, and other maternal factors: A seroepidemiologic study. Am J Obstet Gynecol1987; 156:1137.

44. Weigel, RM, Weigel, MM. Nausea and vomiting of early pregnancy and pregnancy outcome. A meta-analytical review. Br J Obstet Gynaecol 1989; 96: 1312.

45. Boneva, RS, Moore, CA, Botto, L. Nausea during pregnancy and congenital heart defects: a populationbased case-control study. Am J Epidemiol 1999; 149:717.

46. Kallen, B. Hyperemesis gravidarum during pregnancy and delivery. A registry study. In: Nausea and vomiting of pregnancy: state of the art 2000, Koren, G, Bishai, R (Eds), Motherisk, Toronto 2000. P. 36.

47. Seto, a. Einarson, T, Koren, G. Pregnancy outcome following first trimester exposure to antihistamines: meta-analysis. Am J perinat 1997; 174:1565. 